Editorial

\title{
Acknowledgment to Reviewers of Cosmetics in 2020
}

\section{Cosmetics Editorial Office}

Citation: Cosmetics Editorial Office. Acknowledgment to Reviewers of Cosmetics in 2020. Cosmetics 2021, 8,

11. https://doi.org/10.3390/cosmetics 8010011

Published: 25 January 2021

Publisher's Note: MDPI stays neutral with regard to jurisdictional claims in published maps and institutional affiliations.

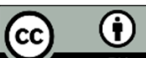

Copyright: (c) 2021 by the authors. Submitted for possible open access publication under the terms and conditions of the Creative Commons Attribution (CC BY) license (http://creativecommons.org/licenses/by/4.0/).

MDPI AG, St. Alban-Anlage 66, 4052 Basel, Switzerland

Peer review is the driving force of journal development, and reviewers are gatekeepers who ensure that Cosmetics maintains its standards for the high quality of its published papers. Thanks to the cooperation of our reviewers, in 2020, the median time to first decision was 13 days and the median time to publication was 34.5 days. The editors would like to express their sincere gratitude to the following reviewers for their precious time and dedication, regardless of whether the papers were finally published:

Amaral, Helena

Annunziata, Giuseppe

Araujo, Andre

Aspatwar, Ashok

Baki, Gabriella

Baran, Anna

Barba, Francisco J.

Barrajon, Enrique

Bartosz, Grzegorz

Baswan, Sudhir

Bellei, Barbara

Berardesca, Enzo

Bertolini, Marta

Bertrand, Martine

Bogdan, Catalina

Bojanowski, K.

Bollini, Flavia

Boyer, Justin G.

Buha, Aleksandra

Burcul, Franko

Callan, Bridgeen

Calvo-Lobo, César

Cantero, Domingo

Caruntu, Constantin

Centini, Marisanna

Chandrasekaran, Murugesan

Coiffard, Laurence J. M.

Conti, Heather

Curcic, Marijana

D'auria, Enza

Da Rosa, Marcelo

De Melo Bonini, Luci Mendes

Del Pozo Bayón, Maria A.

Dong, Qiuchen

Ersilia, Alexa
Esposito, Elisabetta

Filip, Adriana

Fung, Ernest $S$.

Gaweł-Bęben, Katarzyna

Gimenez-Arnau, Elena

Gonda, Sándor

Graf, Christina

Gubitosa, Jennifer

Guerranti, Cristiana

Gupta, Kajal

Guzmán, Eduardo

Gwiazdowska, Daniela

Han, Jaehong

Hano, Christophe

Holefors, Anna

Itou, Takashi

Iwashina, Tsukasa

Jabłońska-Trypuć, Agata

Jaromin, Anna

Juncan, Anca Maria

Kageyama, Hakuto

Kamolz, Lars-Peter

Kim, Hyun Jung

Kim, Ki Hyun

Kim, Ki-Taek

Kim, Kyu-Bong

Kishimoto, Jiro

Klimaszewska, Emilia

Koike, Kenzo

Kojima, Hajime

Koreivienè, Judita

Kottner, Jan

Kung, Chin-Ping

Labrou, Nikolaos

Lagouri, Vasiliki 
Lawrence, Karl

Leclercq, Loïc

Lee, Young-Chul

Lemfack, Marie Chantal

Leon, Lorraine

Lephart, Edwin D.

Lionetti, Nicola

Liu, Chao-Lin

Lodén, Marie

Loglio, Giuseppe

Man, Adrian

Marino, Angela

Marinova, Krastanka

Masters, Eliot

Mazumder, Kishor

Menaa, Farid

Montenegro, Lucia

Montesinos, M. Carmen

Morici, Lisa

Narra, Hema Prasad

Nitulescu, Georgiana

Nunes, Fernando

O'Neill, A. M.

Oancea, Florin

Oliveira, Andreia

Park, Kyoung-Chan

Picca, Anna

Pilmane, Māra

Pratsinis, Harris

Proksch, Ehrhardt

Proverbio, Edoardo

Raeeszadeh-Sarmazdeh, Maryam

Ramanauskienè, Kristina

Rasmussen, Kirsten

Reich, Adam
Renò, Filippo

Ribeiro, Artur

Rodrigues, Francisca

Rodríguez Villanueva, Javier

Rosa, Gonçalo P.

Rosado, Catarina

Ross, Christina L.

Rundo, Leonardo

Sawa, Teiji

Seijas Vázquez, Julio A.

Semenzato, Alessandra

Shim, Kyu-Sik

Sikora, Elżbieta

Slominski, Andrzej

Sowa, Ireneusz

Steel, Christina

Storti, Giuseppe

Strekowski, Lucjan

Sugumaran, Manickam

Svecnjak, Lidija

Takemori, Hiroshi

Tassoni, Annalisa

Tayebati, Seyed Khosrow

Tromelin, Anne

Tungmunnithum, Duangjai

Valacchi, Giuseppe

Varvaresou, Athanasia

Vassallo, Antonio

Villa, Carla

Wei, Kai-Che

Weighardt, Heike

Wińska, Katarzyna

Yang, Chao-Hsun

Yarosh, Daniel B.

Zastrow, Leonhard 